\title{
Pequenos mamíferos (Didelphimorphia, Chiroptera e Rodentia) em egagropilos de Tyto furcata (coruja-das-igrejas) (Aves, Tytonidae) do sul do Brasil
}

\author{
Jorge José Cherem ${ }^{1 *}$ \\ Patrícia Hadler ${ }^{2}$ \\ Narla Shannay Stutz ${ }^{3}$ \\ Ulyses Francisco José Pardiñas ${ }^{4}$ \\ ${ }^{1}$ Caipora Cooperativa \\ Rua Desembargador Vitor Lima, 260/908, CEP 88040-400, Florianópolis - SC, Brasil \\ ${ }^{2}$ Universidade Federal de Santa Catarina, Florianópolis - SC, Brasil \\ ${ }^{3}$ Programa de Pós Graduação em Geociências, Universidade Federal do Rio Grande do Sul \\ Porto Alegre - RS, Brasil \\ ${ }^{4}$ Instituto de Diversidad y Evolución Austral (IDEAus-CONICET), Puerto Madryn - Chubut, Argentina \\ * Autor para correspondência \\ jjcherem@gmail.com
}

Submetido em 30/04/2018

Aceito para publicação em 08/08/2018

\section{Resumo}

A análise e identificação de pequenos mamíferos em egagropilos de coruja, em especial do gênero Tyto, são consideradas uma ferramenta útil e complementar na mastozoologia. No entanto, poucos estudos deste tipo têm sido desenvolvidos no sul do Brasil. Foram analisados os fragmentos cranianos e mandibulares de pequenos mamíferos encontrados em egagropilos de Tyto furcata coletados em sete localidades dos três estados do sul do país. Ao todo, foram contabilizados 2.382 indivíduos pertencentes a 29 táxons, incluindo cinco marsupiais (Didelphidae; 1,39\% dos indivíduos), quatro quirópteros (Molossidae, Phyllostomidae e Vespertilionidae; 0,25\%) e 20 roedores (Cricetidae, Muridae, Caviidae e Echimyidae; 98,36\%). Os roedores Akodon spp., Oligoryzomys sp. e Mus musculus foram os táxons mais comuns. A amostra inclui espécies com poucos registros para o sul do Brasil, como Bibimys sp., Calomys tener e Wilfredomys oenax. A riqueza de pequenos mamíferos obtida nessas amostras corrobora a utilidade e importância da análise de egagropilos de aves de rapina. Ainda assim, são necessários estudos mais detalhados sobre a morfologia e identificação das espécies, como de Akodon e Oligoryzomys, considerando-se a natureza fragmentária do material nos egagropilos.

Palavras-chave: Mus; Oligoryzomys; Paraná; Rio Grande do Sul; Santa Catarina

\section{Abstract}

Small mammals (Didelphimorphia, Chiroptera and Rodentia) in pellets of Tyto furcata (barn owl) (Aves, Tytonidae) from southern Brazil. The study of small mammals in owl pellets, especially of the genus Tyto, is considered a useful and complementary tool in mammalogy. However, few studies using this kind of information have been conducted in southern Brazil. We analyzed craniodental remains of small mammals in Tyto 
furcata pellets from seven locations in the three southern Brazilian states. In all, 2,382 individuals belonging to 29 taxa were recorded, including 5 marsupials (Didelphidae; 1.39\% of individuals), 4 chiropterans (Molossidae, Phyllostomidae and Vespertilionidae; 0.25\%), and 20 rodents (Cricetidae, Muridae, Caviidae and Echimyidae; 98.36\%). The rodents Akodon spp., Oligoryzomys sp. and Mus musculus were the most common taxa. The sample also included taxa with few recorded localities previously published, such as the rodents Bibimys sp., Calomys tener and Wilfredomys oenax. The richness of small mammals obtained in the samples studied corroborates the utility and importance of analyzing pellets of birds of prey. In addition, our contribution highlights the need for more detailed studies on the morphology and identification of several mammalian species, such as those of the genera Akodon and Oligoryzomys, considering the fragmentary nature of the specimens retrieved from pellets.

Key words: Mus; Oligoryzomys; Paraná; Rio Grande do Sul; Santa Catarina

\section{Introdução}

A análise e a identificação de pequenos mamíferos em egagropilos (pelotas) de aves de rapina são uma ferramenta útil em estudos mastozoológicos e permitem complementar as informações obtidas por métodos tradicionais de amostragem (e. g., TAYLOR, 1994; TORRE et al., 2004). Além de contribuir com o levantamento de espécies de pequenos mamíferos em uma determinada área, os dados obtidos podem ser utilizados em estudos paleoambientais, de distribuição geográfica, variações na abundância dos táxons, morfologia dentária, entre outros (VOGLINO et al., 2004; TETA et al., 2010; MEEK et al., 2012; HADLER et al., 2016).

Dentre as aves de rapina, as corujas do gênero Tyto têm sido particularmente estudadas em várias partes do mundo quanto a esses aspectos (e. g., ANDERSON; LONG, 1961; MASSOIA, 1983; MOTTA-JÚNIOR; TALAMONI, 1996; BELLOCQ, 1998; LEKUNZE et al., 2001; BONVICINO; BEZERRA, 2003; PARDIÑAS et al., 2005; CHARTER et al., 2007; SOUZA et al., 2010; BRITO et al., 2015). Essas corujas são quase cosmopolitas e habitam principalmente áreas abertas, inclusive ambientes antrópicos. São predominantemente noturnas, alimentando-se de pequenos vertebrados e insetos, que são capturados na maioria dos ambientes presentes em sua área de forrageamento (área de vida média $=6,82 \mathrm{~km}^{2}$ ). Nidificam em cavernas e construções humanas, o que permite que o material regurgitado se preserve por muito tempo (SICK, 2001; HEISLER et al., 2016).

Para o sul do Brasil, os poucos estudos realizados com pelotas de Tyto furcata (Temminck, 1827) têm corroborado seu potencial para o registro de pequenos mamíferos, seja pelo grande número de espécies encontradas ou pelos táxons pouco conhecidos regionalmente (e. g., GONZÁLEZ et al., 1999; SCHEIBLER; CHRISTOFF, 2007; PETERS et al., 2013).

Neste estudo, nós analisamos os fragmentos cranianos e mandibulares de pequenos mamíferos encontrados em egagropilos de T. furcata coletados em sete localidades no sul do Brasil. Com isto, temos por objetivo contribuir para o conhecimento das assembleias atuais de pequenos mamíferos da porção subtropical da Mata Atlântica, incluindo o registro de táxons pouco conhecidos.

\section{Material e Métodos}

Os egagropilos estudados foram coletados dentro e ao redor de construções humanas (áreas cobertas, galpões, igrejas e casas abandonadas) utilizadas como abrigo por Tyto furcata em sete localidades nos três estados da região Sul do Brasil (Tabela 1; Figura 1). Essas localidades abrangem regiões fitoecológicas interioranas (Floresta Ombrófila Mista e Floresta Estacional) e litorâneas (Floresta Ombrófila Densa), inseridas no bioma Mata Atlântica (LEITE, 2002).

As amostras de cada localidade foram depositadas por lote na Coleção de Fragmentos Ósseos do Laboratório de Paleontologia (UFSC-CF), Centro de Filosofia e Ciências Humanas, Universidade Federal de Santa Catarina, Florianópolis, estado de Santa Catarina (Tabela 1). 
TABELA 1: Localidades de registro de egagropilos de Tyto furcata nos estados do Paraná (PR), Santa Catarina (SC) e Rio Grande do Sul (RS), sul do Brasil. UFSC-CF = Coleção de Fragmentos Ósseos do Laboratório de Paleontologia da UFSC.

\begin{tabular}{lccccc}
\hline Localidade & Sigla & Altitude & Coordenadas (WGS 84) & Data & № tombo \\
\hline Floresta Ombrófila Mista (FOM) & & & & & \\
\hline São Mateus do Sul/PR & SMS & $800 \mathrm{~m}$ & $25^{\circ} 50^{\prime} 55^{\prime \prime}$ S, $50^{\circ} 24^{\prime} 58^{\prime \prime} \mathrm{W}$ & set/2014 a ago/2015 & UFSC-CF 08 \\
Lages/SC & LAG & $870 \mathrm{~m}$ & $27^{\circ} 46^{\prime} 24^{\prime \prime} \mathrm{S}, 50^{\circ} 32^{\prime} 17^{\prime \prime} \mathrm{W}$ & out/2014 & UFSC-CF 05 \\
\hline
\end{tabular}

Ecótono Floresta Ombrófila Mista - Floresta Estacional (ECO)

\begin{tabular}{llllll}
\hline Usina Hidrelétrica Quebra Queixo/SC & QQ & $550 \mathrm{~m}$ & $26^{\circ} 37^{\prime} 11^{\prime}$ 'S, $52^{\circ} 33^{\prime} 03^{\prime}$ 'W & set-dez/2016 & UFSC-CF 23
\end{tabular}

Floresta Estacional (FES)

\begin{tabular}{llllll}
\hline Alpestre/RS & ALP & $240 \mathrm{~m}$ & $27^{\circ} 08^{\prime} 57^{\prime}{ }^{\prime} \mathrm{S}, 53^{\circ} 03^{\prime} 42^{\prime \prime} \mathrm{W}$ & set $/ 2015$ & UFSC-CF 06
\end{tabular}

Floresta Ombrófila Densa (FOD)

\begin{tabular}{lccccc}
\hline Garuva/SC & GAR & $10 \mathrm{~m}$ & $25^{\circ} 59^{\prime} 44^{\prime \prime} \mathrm{S}, 48^{\circ} 47^{\prime} 20^{\prime \prime} \mathrm{W}$ & set/2012 a abr/2013 & UFSC-CF 07 \\
Araquari/SC & ARA & $5 \mathrm{~m}$ & $26^{\circ} 29^{\prime} 50^{\prime \prime} \mathrm{S}, 48^{\circ} 45^{\prime} 15^{\prime \prime} \mathrm{W}$ & jul-ago/2013 & UFSC-CF 10 \\
Siderópolis/SC & SID & $150 \mathrm{~m}$ & $28^{\circ} 35^{\prime} 58^{\prime \prime} \mathrm{S}, 49^{\circ} 33^{\prime} 24^{\prime \prime} \mathrm{W}$ & jul/2003 & UFSC-CF 04 \\
\hline
\end{tabular}

FIGURA 1: Localização dos sítios de coleta de egagropilos de Tyto furcata na região Sul do Brasil. Siglas das localidades conforme Tabela 1. Mapa modificado a partir de MMA (1992).

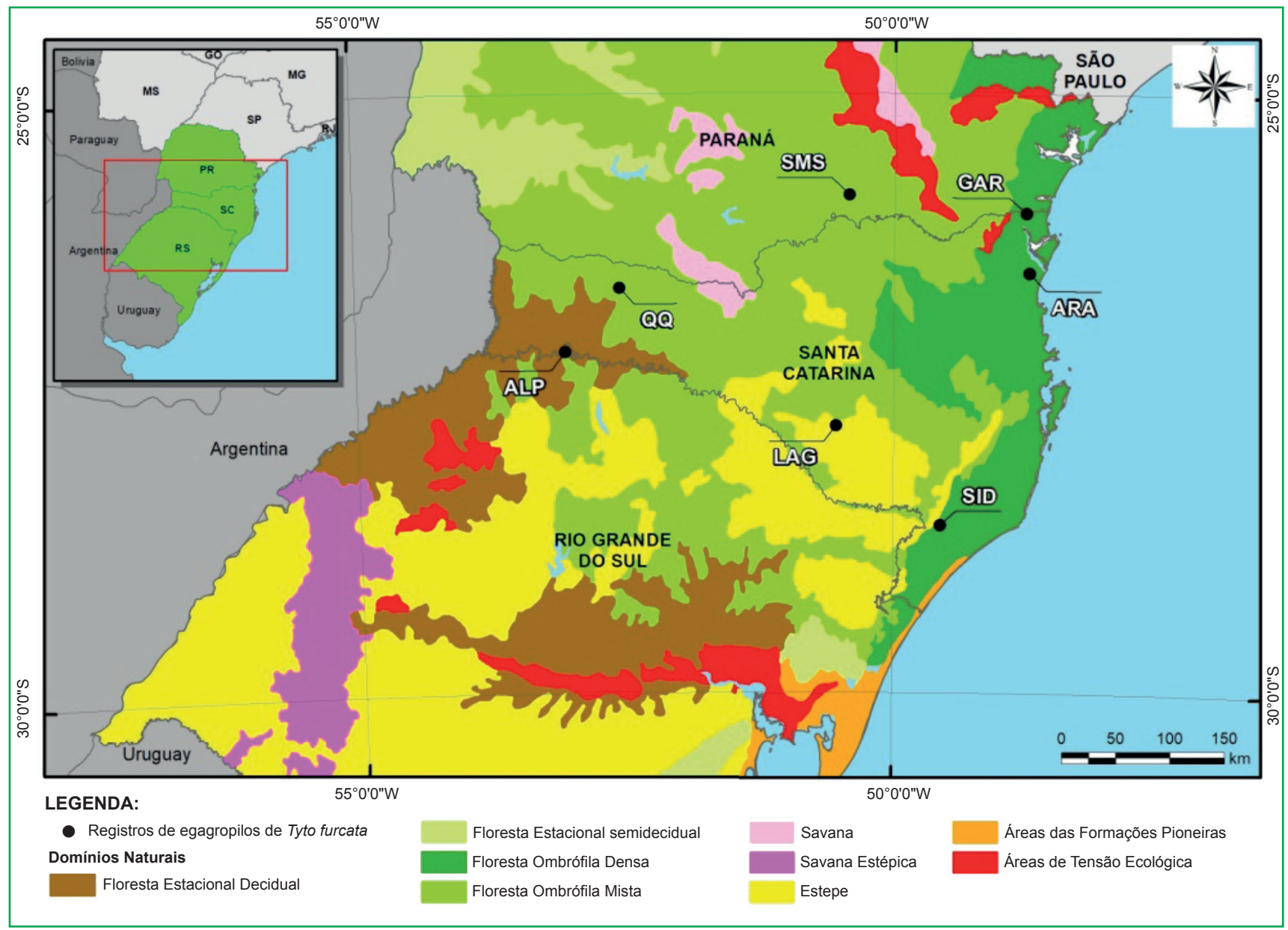


A identificação dos pequenos mamíferos foi feita por comparação com material depositado na Coleção de Mamíferos do Departamento de Ecologia e Zoologia da UFSC (Anexo I) e com base nos trabalhos de Barquez et al. (1999), Gonçalves et al. (2007), Gardner (2008), Quintela et al. (2014), Patton et al. (2015), Hadler et al. (2016) e Stutz et al. (2017). Os caracteres diagnósticos que permitiram a identificação dos gêneros Akodon, Bibimys, Calomys, Juliomys, Oligoryzomys, Oxymycterus, Sooretamys e Cavia, e das espécies, Necromys lasiurus, Sooretamys angouya, Wilfredomys oenax e Euryzygomatomys spinosus foram apresentados em Hadler et al. (2016) e Stutz et al. (2017); e para os gêneros de quirópteros, em Barquez et al. (1999). Os caracteres diagnósticos para os demais gêneros e espécies são comentados nos resultados.

O número mínimo de indivíduos (MNI) por espécie e localidade foi determinado pela contagem das mandíbulas ou maxilares direitos ou esquerdos, considerando-se o maior número para cada táxon por localidade (BEISAW, 2013).

A nomenclatura e o ordenamento taxonômico seguem Gardner (2008), para Didelphimorphia e Chiroptera, Wilson e Reeder (2005), para Muridae, e Patton et al. (2015), para os demais roedores. São indicadas as espécies ameaçadas em nível global (IUCN, 2017), nacional (MMA, 2014) e estadual (PARANÁ, 2010; SANTA CATARINA, 2011; RIO GRANDE DO SUL, 2014), assim como as espécies exóticas invasoras listadas em Paraná (2015), Santa Catarina (2012) e Rio Grande do Sul (2013).

Para estimar o número de espécies ocorrentes em cada amostra, foi utilizado o estimador de riqueza baseado em abundância Chao 1 com 1.000 aleatorizações (GOTELLI; COLWELL, 2011). Os quirópteros não foram incluídos nesta análise, pois tornam a estimativa menos precisa por serem pouco frequentes nas amostras (LEMOS et al., 2015).

\section{Resultados}

Nas amostras de egagropilos de Tyto furcata provenientes das sete localidades no sul do Brasil foram registrados 2.382 indivíduos, representando 29 táxons de pequenos mamíferos de nove famílias e três ordens. As amostras incluem cinco táxons de Didelphimorphia (família Didelphidae), quatro de Chiroptera (Molossidae, Phyllostomidae e Vespertilionidae) e 20 de Rodentia (Cricetidae, Muridae, Caviidae e Echimyidae) (Tabela 2; Figura 2).

TABELA 2: Número mínimo de indivíduos por táxon de pequenos mamíferos registrado em egagropilos de Tyto furcata provenientes de sete localidades na região Sul do Brasil. Siglas das regiões fitoecológicas e localidades conforme Tabela 1.

\begin{tabular}{|c|c|c|c|c|c|c|c|c|c|}
\hline \multirow{2}{*}{ Táxon } & \multicolumn{2}{|c|}{ FOM } & \multirow{2}{*}{$\begin{array}{c}\text { ECO } \\
Q Q \\
\end{array}$} & \multirow{2}{*}{$\begin{array}{l}\text { FES } \\
\text { ALP } \\
\end{array}$} & \multicolumn{3}{|c|}{ FOD } & \multirow{2}{*}{ Total } & \multirow{2}{*}{$\%$} \\
\hline & SMS & LAG & & & GAR & ARA & SID & & \\
\hline \multicolumn{10}{|l|}{ Didelphimorphia } \\
\hline \multicolumn{10}{|l|}{ Didelphidae } \\
\hline Cryptonanus guahybae (Tate, 1931) & 1 & 4 & 4 & 1 & 2 & 1 & 1 & 14 & 0,59 \\
\hline Gracilinanus microtarsus (Wagner, 1842) & 2 & 1 & 2 & & 3 & & & 8 & 0,34 \\
\hline Marmosa paraguayana Tate, 1931 & & & & & 1 & & & 1 & 0,04 \\
\hline Monodelphis sp. & 2 & & 3 & & 2 & & & 7 & 0,29 \\
\hline Didelphidae gen. et sp. indet. & & 3 & & & & & & 3 & 0,13 \\
\hline \multicolumn{10}{|l|}{ Chiroptera } \\
\hline \multicolumn{10}{|l|}{ Molossidae } \\
\hline Molossus molossus (Pallas, 1766) & & & & & & 1 & & 1 & 0,04 \\
\hline Phyllostomidae & & & & & & & & & \\
\hline
\end{tabular}




\begin{tabular}{|c|c|c|c|c|c|c|c|c|c|}
\hline \multirow{2}{*}{ Táxon } & \multicolumn{2}{|c|}{ FOM } & \multirow{2}{*}{\begin{tabular}{|c|} 
ECO \\
QQ \\
\end{tabular}} & \multirow{2}{*}{$\begin{array}{l}\text { FES } \\
\text { ALP }\end{array}$} & \multicolumn{3}{|c|}{ FOD } & \multirow{2}{*}{ Total } & \multirow{2}{*}{$\%$} \\
\hline & SMS & LAG & & & GAR & ARA & SID & & \\
\hline \multicolumn{10}{|l|}{ Vespertilionidae } \\
\hline Eptesicus diminutus Osgood, 1915 & & & & & & 3 & & 3 & 0,13 \\
\hline Myotis sp. & & & & & & 1 & & 1 & 0,04 \\
\hline \multicolumn{10}{|l|}{ Rodentia } \\
\hline \multicolumn{10}{|l|}{ Cricetidae } \\
\hline Akodon spp. & 81 & 28 & 119 & 14 & 7 & 1 & 5 & 255 & 10,71 \\
\hline Bibimys sp. & 2 & 8 & 3 & & & & & 13 & 0,55 \\
\hline Brucepattersonius sp. & 1 & & 2 & 3 & & & 2 & 8 & 0,34 \\
\hline Calomys tener (Winge, 1887) & 31 & 15 & 12 & 4 & & & & 62 & 2,60 \\
\hline Euryoryzomys russatus (Wagner, 1848) & & & & & 5 & & & 5 & 0,21 \\
\hline Juliomys pictipes (Osgood, 1933) & & & & & 9 & & & 9 & 0,38 \\
\hline Juliomys sp. & & 1 & & & & & & 1 & 0,04 \\
\hline Necromys lasiurus (Lund, 1840) & 4 & 6 & 12 & 8 & & & & 30 & 1,26 \\
\hline Nectomys squamipes (Brants, 1827) & & 2 & & & 9 & 1 & 1 & 13 & 0,55 \\
\hline Oligoryzomys sp. & 161 & 515 & 61 & 43 & 229 & 153 & 30 & 1.192 & 50,04 \\
\hline Oxymycterus nasutus (Waterhouse, 1837) & 3 & 8 & 3 & & & & & 14 & 0,59 \\
\hline Oxymycterus sp. & 1 & 1 & 1 & & 6 & & 2 & 11 & 0,46 \\
\hline Scapteromys meridionalis Quintela et al., 2014 & & 3 & 2 & & & & & 5 & 0,21 \\
\hline Sooretamys angouya (G. Fischer, 1814) & 4 & 22 & 2 & & 1 & & & 29 & 1,22 \\
\hline Wilfredomys oenax (Thomas, 1928) & 2 & & & & & & & 2 & 0,08 \\
\hline \multicolumn{10}{|l|}{ Muridae } \\
\hline Mus musculus Linnaeus, 1758 & 26 & & 153 & 4 & 19 & 470 & & 672 & 28,21 \\
\hline Rattus norvegicus (Berkenhout, 1769) & & & & 1 & 2 & 8 & & 11 & 0,46 \\
\hline Rattus rattus (Linnaeus, 1758) & 1 & 4 & 3 & 1 & & & & 9 & 0,38 \\
\hline \multicolumn{10}{|l|}{ Caviidae } \\
\hline Cavia sp. & & & & 1 & & & & 1 & 0,04 \\
\hline \multicolumn{10}{|l|}{ Echimyidae } \\
\hline Euryzygomatomys spinosus (G. Fischer, 1814) & & & 1 & & & & & 1 & 0,04 \\
\hline Total de indivíduos & 323 & 621 & 383 & 80 & 295 & 639 & 41 & 2.382 & 100,00 \\
\hline Riqueza (total de pequenos mamíferos) & 16 & 15 & 16 & 10 & 13 & 9 & 6 & 29 & \\
\hline Riqueza (não voadores) & 15 & 15 & 16 & 10 & 13 & 6 & 6 & 25 & \\
\hline Estimativa de riqueza (não voadores; Chao 1) & 16 & 16,5 & 16 & 16 & 13 & 9 & 6 & & \\
\hline
\end{tabular}


FIGURA 2: A-I, vista lateral das mandíbulas de: A, Cryptonanus guahybae; B, Gracilinanus microtarsus; C, Marmosa paraguayana; D, Didelphidae indeterminado; E, Monodelphis sp.; F, Wilfredomys oenax; G, Euryoryzomys russatus; H, Scapteromys meridionalis; I, Euryzygomatomys spinosus. J-D', vista ventral dos fragmentos dos crânios de: J, Molossus molossus; K, Artibeus lituratus; L, Eptesicus diminutus; M, Myotis sp.; N, Akodon montensis; O, Akodon paranaensis; P, Bibimys sp.; Q, Brucepattersonius sp.; R, Calomys tener; S, Juliomys pictipes; T, Juliomys sp.; U, Necromys lasiurus; V, Nectomys squamipes; W, Oligoryzomys sp.; X, Oxymycterus nasutus; Y, Oxymycterus sp.; Z, Sooretamys angouya; A', Mus musculus; B', Rattus rattus; C', Rattus norvegicus; D', Cavia sp. Escala $=1 \mathrm{~mm}$.

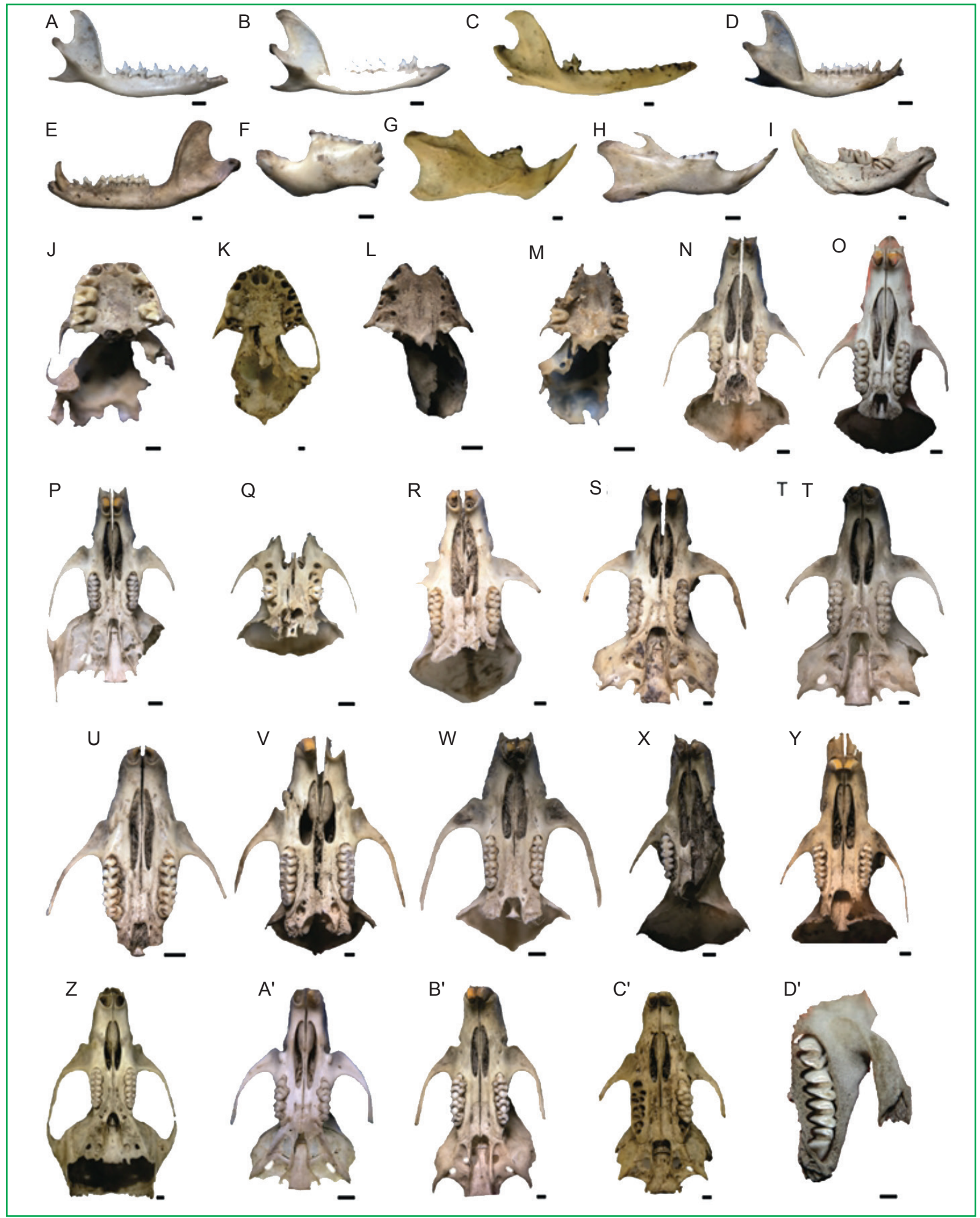




\section{Composição das amostras}

As amostras foram predominantemente compostas por roedores (70\% dos táxons e 98,36\% dos indivíduos) e, dentre esses, pela família Cricetidae (53,33\% e 69,24\%, respectivamente). Os marsupiais compreenderam cinco táxons e 1,39\% do total de indivíduos e os quirópteros, quatro e $0,25 \%$, respectivamente. Cryptonanus guahybae, Akodon spp. e Oligoryzomys sp. foram registrados nas sete localidades. Por outro lado, 12 táxons ocorreram em apenas uma localidade, como Marmosa paraguayana, Molossus molossus e Euryoryzomys russatus. Oligoryzomys sp. representou cerca de 50\% do total de indivíduos, sendo o táxon mais frequente em cinco das sete localidades. Em seguida, aparecem Mus musculus (28,21\%; mais frequente em Quebra Queixo e Araquari) e Akodon spp. (10,71\%). Os outros 26 táxons em conjunto perfizeram $11,04 \%$.

Wilfredomys oenax, espécie registrada em São Mateus do Sul, é considerada ameaçada de extinção, listada como em perigo (EN) em nível global, nacional e para o estado do Rio Grande do Sul, e como criticamente em perigo (CR) para o estado do Paraná. Três roedores da família Muridae (Mus musculus, Rattus norvegicus e $R$. rattus) foram registrados nos egagropilos e estão nas listas de espécies exóticas invasoras do Paraná e Santa Catarina. Mus musculus também é listado como exótico invasor para o Rio Grande do Sul. Exceto para Siderópolis, pelo menos uma dessas espécies foi identificada em cada localidade, e o maior número de roedores exóticos, M. musculus em particular, foi obtido em Araquari (73,55\%).

O maior número de táxons foi registrado em São Mateus do Sul e Quebra Queixo $(\mathrm{n}=16)$, seguido por Lages $(n=15)$. Essas três localidades situam-se em Floresta Ombrófila Mista ou no ecótono entre esta região fitoecológica e a Floresta Estacional Decidual. Por outro lado, os menores números foram registrados em Araquari $(n=9)$ e Siderópolis $(n=6)$, ambas em Floresta Ombrófila Densa.

As estimativas de riqueza de marsupiais e roedores, obtidas pelo estimador Chao 1 , indicam que os valores obtidos estão próximos ou foram iguais ao estimado, exceto em Alpestre (10 espécies registradas e 16 estimadas) e Araquari (6 e 9, respectivamente) (Tabela 2).

Quatro espécies de área aberta ou alterada (Bibimys sp., Calomys tener, Necromys lasiurus e Oxymycterus nasutus) e uma espécie exótica (Rattus rattus) foram registradas em pelo menos três das quatro localidades do interior (em Floresta Ombrófila Mista ou Floresta Estacional Decidual), mas não estiveram presentes nas amostras da Floresta Ombrófila Densa.

\section{Identificação dos táxons}

\section{Família Didelphidae}

Dentre os marsupiais didelfídeos, Marmosa paraguayana distingue-se dos demais táxons por suas maiores dimensões, fenestra maxilopalatina muito estreita e fenestra maxilar ausente (VOSS; JANSA, 2009).

O gênero Monodelphis apresenta morfologia das fenestras palatais similar à Marmosa, diferenciando-se principalmente por suas menores dimensões, ausência da cúspide estilar $\mathrm{C}$ nos molares superiores, cingulídeo amplo e talonídeo curto nos molares inferiores (GOIN; REY, 1997; VOSS; JANSA, 2009). No entanto, a identificação das espécies de Monodelphis a partir dos fragmentos encontrados requer um estudo mais aprofundado de sua morfologia. Com base na distribuição atualmente conhecida, M. dimidiata (Wagner, 1847) é a única espécie registrada para São Mateus do Sul e Quebra Queixo (PAVAN; VOSS, 2016), enquanto $M$. americana (Müller, 1776), M. iheringi (Thomas, 1888) e M. scalops (Thomas, 1888) são de possível ocorrência em Garuva (GARDNER, 2008; PAVAN; VOSS, 2016).

Gracilinanus microtarsus distingue-se de Cryptonanus guahybae pela presença de fenestras palatais, segundo e terceiro molares superiores subiguais em altura e segundo molar inferior mais alto do que o terceiro (VOSS; JANSA, 2009). Além disso, observouse que G. microtarsus apresenta uma série molar inferior comparativamente mais longa e um cíngulo labial completo nos três primeiros molares inferiores (geralmente incompleto no $\mathrm{m} 2$ e $\mathrm{m} 3$ de $C$. guahybae). 
Ambos apresentam cíngulo anterior completo no terceiro molar superior, sendo em geral mais amplo em $G$. microtarsus.

Um táxon de Didelphidae registrado em Lages não pôde ser identificado. A amostra referida a este táxon inclui três maxilares direitos, dois maxilares esquerdos e dois pares de mandíbulas. Distingue-se dos demais didelfídeos pela seguinte combinação de caracteres: fenestra maxilar pequena, fenestra palatina ausente, molares superiores mais comprimidos anteroposteriormente, segundo prémolar inferior maior do que o terceiro, e entoconídeo e hipoconulídeo subiguais em altura nos três primeiros molares inferiores.

\section{Família Molossidae}

O gênero Molossus está representado por duas espécies no sul do Brasil, M. rufus E. Geoffroy, 1805 e M. molossus (Pallas, 1766), sendo esta de menor porte (GARDNER, 2008; MIRANDA et al., 2011). $\mathrm{O}$ indivíduo registrado em Araquari foi referido a $M$. molossus por apresentar comprimento da mandíbula $=11,58 \mathrm{~mm}$, condizente com as dimensões apresentadas por Barquez (1983) para M. molossus, além de possuir uma crista sagital pouco desenvolvida, característica da espécie.

\section{Família Phyllostomidae}

Entre as espécies do gênero Artibeus reconhecidas para o sul do Brasil (GARDNER, 2008; MIRANDA et al., 2011), o indivíduo encontrado em São Mateus do Sul foi identificado como A. lituratus por apresentar constrição pós-orbital estreita (largura $=6,9 \mathrm{~mm}$ ), processos orbitais bem desenvolvidos e ausência do M3, além de seu tamanho grande (BARQUEZ et al., 1999).

\section{Família Vespertilionidae}

Para a região Sul do Brasil, quatro espécies do gênero Eptesicus foram reconhecidas por Miranda et al. (2011): E. brasiliensis (Desmarest, 1819), E. diminutus Osgood, 1915, E. furinalis (d'Orbigny, 1847) e E. taddei Miranda et al. 2006. As duas mandíbulas registradas em Araquari apresentam comprimento total $=9,6$ e 9,7 $\mathrm{mm}$ e comprimento da série dentária $=5,3$ e $5,4 \mathrm{~mm}$.
Essas medidas estão dentro da variação registrada para E. diminutus conforme Barquez et al. (1999).

O gênero Myotis pode ser diagnosticado pela presença de três pré-molares entre os Vespertilionidae. Para Santa Catarina, pelo menos seis espécies do gênero são reconhecidas: M. albescens (E. Geoffroy, 1806); M. dinelli Thomas, 1902; M. levis (I. Geoffroy, 1824); M. nigricans (Schinz, 1821); M. riparius Handley, 1960 e M. ruber (E. Geoffroy, 1806) (PASSOS et al., 2010). Dentre essas, apenas M. levis e M. nigricans não possuem crista sagital (BARQUEZ et al., 1999), assim como o espécime estudado, proveniente de Araquari. No entanto, não há caracteres suficientes preservados para permitir sua identificação específica.

\section{Família Cricetidae}

O gênero Akodon, presente em egagropilos de todas as localidades, inclui duas espécies de ocorrência confirmada para Quebra Queixo (CHEREM et al., 2008), São Mateus do Sul e Alpestre (dados não publicados), identificadas a partir de seu número diploide: A. montensis Thomas, $1913(2 \mathrm{n}=24-26)$ e A. paranaensis Christoff et al., $2000(2 \mathrm{n}=44)$. Conforme sugerido por Gonçalves et al. (2007), a relação entre o comprimento da série molar superior com a constrição interorbital permite distinguir em grande parte essas espécies. A comparação dos valores daqueles espécimes cariotipados com espécimes de Akodon em egagropilos de São Mateus do Sul, Lages, Alpestre e Quebra Queixo, nos quais foi possível tomar essas medidas, sugere a ocorrência de ambas espécies nessas amostras (Figura 3).

Para os maxilares isolados que apresentavam os três molares, foram registrados os seguintes intervalos de comprimento da série molar: São Mateus do Sul = 3,85$4,8 \mathrm{~mm}(\mathrm{n}=24)$; Lages $=3,9-4,45 \mathrm{~mm}(\mathrm{n}=5)$; Quebra Queixo $=4,1-4,7 \mathrm{~mm}(\mathrm{n}=37)$; Garuva $=4,25-4,3 \mathrm{~mm}$ $(\mathrm{n}=2)$; e Araquari $=4,35 \mathrm{~mm}(\mathrm{n}=1)$.

Essas medidas em conjunto sugerem que tanto A. montensis quanto $A$. paranaensis estão presentes nas amostras de São Mateus do Sul, Lages e Quebra Queixo e pelo menos a primeira em Alpestre. Em Garuva e Araquari, as medidas das séries molares sugerem que se tratam de $A$. montensis. No entanto, em 
FIGURA 3: Comparação entre os valores de comprimento da série molar superior e constrição interorbital, em milímetros, para amostras de Akodon em egagropilos de Tyto furcata (SMS, LAG, ALP e QQ) e espécimes de Akodon montensis e A. paranaensis da região Sul do Brasil. Siglas das localidades conforme Tabela 1.

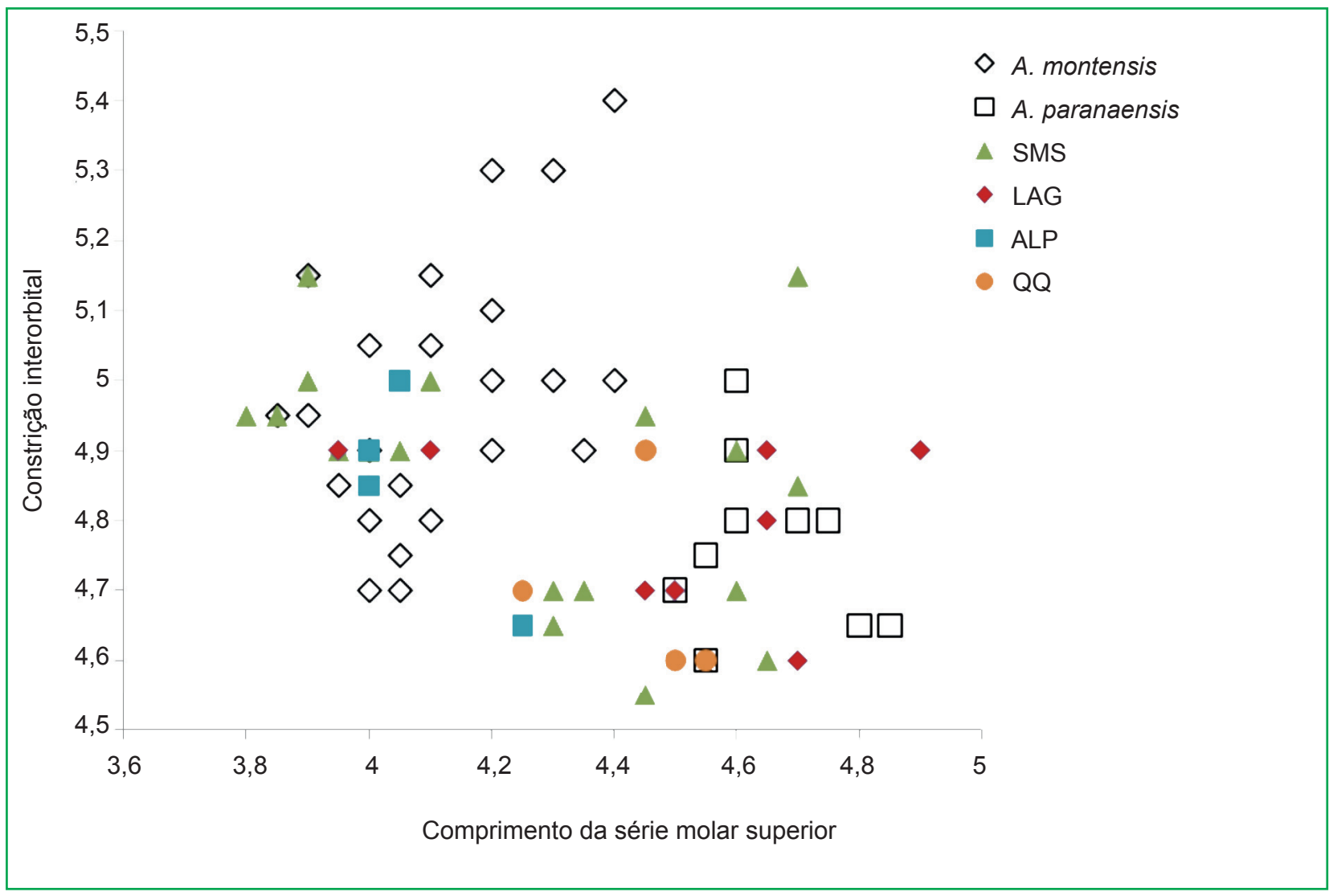

função da similaridade entre essas espécies e do estado fragmentário das amostras, com muitos espécimes com a séria dentária incompleta, o material é referido como Akodon spp.

A taxonomia alfa do akodontino Bibimys é controversa. Para o Brasil, apenas uma espécie tem sido referida, Bibimys labiosus (Winge, 1887), cuja localidade tipo é Lagoa Santa, estado de Minas Gerais. No entanto, para o Paraguai e a província de Misiones, Argentina, as populações de Bibimys são assinaladas a B. chacoensis (Shamel, 1931). O status taxonômico dessas duas formas nominais é incerto (PARDIÑAS et al., 2017) e, no momento, não é possível assinalar confiavelmente os animais do sul do Brasil a uma espécie em particular.

O gênero Brucepattersonius apresenta uma morfologia craniana e dentária similar a Oxymycterus, particularmente com $O$. nasutus, por seu pequeno porte (MASSOIA, 1963). A partir da comparação com espécimes depositados na coleção da UFSC, os fragmentos nos egagropilos foram atribuídos a Brucepattersonius por apresentar forâmen incisivo mais curto, placa zigomática menos inclinada posteriormente e com uma pequena tuberosidade na base, pequena fossa no maxilar, anterior ao M1, mais marcada, raiz acessória labial no M1 (rara em O. nasutus), mandíbula mais curta, fossa retromolar em geral mais profunda e processo coronoide mais alto e menos inclinado posteriormente.

Com base em caracteres moleculares, Dias (2016) registrou duas espécies de Brucepattersonius para localidades próximas aos pontos de coleta dos egagropilos aqui analisados: B. soricinus Hershkovitz, 1998 para São Mateus do Sul e B. iheringi (Thomas, 1896) para as demais localidades. No entanto, não são conhecidos caracteres que permitam confirmar a identificação dessas espécies a partir dos fragmentos presentes nos egagropilos. Além disso, o status 
taxonômico e os limites de distribuição de outras três espécies de Misiones, Argentina, são desconhecidos (VILELA et al., 2015). Desta forma, os espécimes em egagropilos não foram identificados em nível de espécie.

Para o gênero Calomys, registrado em quatro localidades (São Mateus do Sul, Lages, Quebra Queixo e Alpestre), duas espécies são conhecidas para o sul do Brasil, Calomys laucha (G. Fischer, 1814) e $C$. tener (Winge, 1887) (SALAZAR-BRAVO, 2015). Os espécimes foram referidos a $C$. tener por apresentarem margem supraorbital distintamente saliente, projeção capsular conspícua e m3 em forma de "S", conforme Salazar-Bravo (2015).

Entre o material de Juliomys, as maiores dimensões e a ausência da depressão esquamosal-alisfenoide permitiram atribuir os espécimes de Garuva a J. pictipes. Por outro lado, as dimensões menores e a ausência dessa depressão indicam que os espécimes de Lages possam se referir a J. ossitenuis Costa et al. 2007 ou a J. ximenezi Christoff et al. 2016. Essas duas espécies ocorrem na Floresta Ombrófila Mista do sul do Brasil, mas sua diferenciação baseia-se principalmente em dados cariotípicos ou moleculares (PARDIÑAS et al., 2008; CHRISTOFF et al., 2016). Desta forma, os espécimes de Lages são tratados como Juliomys sp.

O gênero Oligoryzomys, registrado em todas as localidades, inclui duas espécies no sul do Brasil, O. flavescens (Waterhouse, 1837) e O. nigripes (Olfers, 1818), muito similares quanto à morfologia craniana. Langguth (1963) reportou diferenças nas medidas cranianas para as populações uruguaias dessas espécies, incluindo o comprimento da série molar superior, $O$. flavescens entre 3,2 e 3,9 $\mathrm{mm}$ e $O$. nigripes $(=$ O. delticola) entre 3,9 e 4,6 mm. No entanto, Machado et al. (2011) obtiveram valores menores e maior sobreposição para populações brasileiras das mesmas espécies (O. flavescens com intervalo entre 3,01 e 3,58 $\mathrm{mm}$ e $O$. nigripes entre 3,06 e 4,28 mm). Alguns espécimes de $O$. nigripes presentes na coleção da UFSC e identificados por cariótipo $(2 n=62)$ também possuem valores de comprimento da série molar superior (entre 3,1 e 3,7 mm) menores do que as populações uruguaias. Outros caracteres considerados diagnósticos para essas duas espécies, como o comprimento do forâmen incisivo, a posição do forâmen frontal (ou suprafrontal) e o desenvolvimento da projeção capsular na mandíbula (MACHADO et al., 2011; BORONI et al., 2015), mostraram-se variáveis entre os espécimes utilizados para comparação. Dessa forma, e considerando o estado fragmentário do material presente nos egagropilos, os espécimes foram referidos como Oligoryzomys sp.

Os espécimes de Oxymycterus nos egagropilos apresentam duas classes de tamanho. Com base na distribuição geográfica (OLIVEIRA; GONÇALVES, 2015), os espécimes pequenos foram referidos a O. nasutus (Waterhouse, 1837). Os espécimes maiores podem se referir a $O$. quaestor Thomas, 1903, que possui ampla distribuição no sul do Brasil, ou a O. dasytrichus (Schinz, 1821), que ocorre no Paraná e nordeste de Santa Catarina (OLIVEIRA; GONÇALVES, 2015; PEÇANHA et al., 2016). No entanto, o estado fragmentário das amostras obtidas nos egagropilos não permite verificar os caracteres diagnósticos dessas duas espécies.

Scapteromys meridionalis foi registrado em Lages e Quebra Queixo. Esta é a menor espécie do gênero e a única com ocorrência conhecida na área abrangida pelas sete localidades analisadas neste estudo (QUINTELA et al., 2014).

As três espécies de sigmodontinos de maior porte foram os Oryzomyini Euryoryzomys russatus (Garuva), Nectomys squamipes (Lages, Garuva, Araquari e Siderópolis) e Sooretamys angouya (São Mateus do Sul, Lages, Quebra Queixo e Garuva). Nectomys squamipes é a maior delas e apresenta forâmen incisivo curto. Euryoryzomys russatus é a menor e distingue-se de S. angouya por apresentar crista supraorbital (PATTON et al., 2015).

\section{Família Muridae}

A família Muridae inclui três espécies de roedores exóticos invasores na América do Sul, caracterizados por apresentarem três séries longitudinais de cúspides nos molares superiores (MOOJEN, 1952). Mus musculus é uma das menores espécies de roedores encontrados nos egagropilos. Possui forâmen incisivo muito longo, fossa mesopterigoidea estreita, tuberosidade marcada na base 
da placa zigomática, mandíbula com crista massetérica ventral longa e conspícua e processo capsular pouco desenvolvido.

As espécies de Rattus que ocorrem no Brasil apresentam porte muito maior que Mus. Rattus rattus apresenta uma cúspide anterolabial menos reduzida no M1 (MOOJEN, 1952) e uma pequena cúspide labial posterior (sensu MUSSER, 1981) independente no m2, a qual está fusionada ao hipoconídeo em $R$. norvegicus. Essas duas espécies diferenciam-se também pela morfologia do teto craniano (MASSOIA; FORNES, 1969).

\section{Família Caviidae}

O gênero Cavia foi encontrado na amostra de Alpestre, representado por um maxilar esquerdo e duas mandíbulas, uma direita e outra esquerda. $\mathrm{O}$ comprimento da série molar superior ao nível alveolar $=8,90 \mathrm{~mm}$ e o fato de o último molar estar em erupção indicam tratar-se de um espécime jovem (idade I) de Cavia aperea Erxleben, 1777 ou C. fulgida Wagler, 1831. Em função da idade e de a fenda terciária externa no terceiro molar superior ainda não estar formada, não é possível determinar a espécie (XIMENEZ, 1980; CHEREM; FERIGOLO, 2012).

\section{Família Echimyidae}

Dois fragmentos de maxilares, um direito e outro esquerdo, e uma mandíbula direita de Euryzygomatomys spinosus foram registrados em Quebra Queixo. A mandíbula apresenta apenas os três primeiros molariformes, com o segundo molar não eclodido, indicando tratar-se de um espécime jovem.

\section{Discussão}

A importância da análise de egagropilos para o estudo de pequenos mamíferos, em particular de corujas do gênero Tyto, tem sido demonstrada por trabalhos desenvolvidos em diversas regiões do mundo. Esse tipo de análise fornece o registro de pequenos mamíferos raros ou difíceis de capturar com armadilhas, além de apresentar baixo custo em ambientes extensos (MORENO; BARBOSA, 1992; FORMOSO et al., 2016; HEISLER et al., 2016), o que também é corroborado no presente estudo. Para uma das localidades analisadas (Quebra Queixo), por exemplo, Cherem et al. (2008) capturaram 15 espécies com um esforço amostral de 5.690 armadilhas-noite, enquanto 16 espécies de pequenos mamíferos não voadores foram registradas nos egagropilos (presente estudo). Dentre as espécies amostradas, Didelphis aurita (WiedNeuwied, 1826), Philander frenatus (Olfers, 1818), Guerlinguetus brasiliensis (Thomas, 1901), Nectomys squamipes e Rattus norvegicus foram registradas apenas nas armadilhas (CHEREM et al., 2008), enquanto Cryptonanus guahybae, Bibimys sp., Brucepattersonius sp., Calomys tener e Oxymycterus nasutus foram exclusivas dos egagropilos. Deve-se considerar, ainda, que os gêneros Bibimys e Calomys contam com poucos registros no sul do país (PATTON et al., 2015).

Tyto furcata ocupa e forrageia em áreas abertas e antropizadas, sendo considerada uma predadora especialista na captura de roedores (ANDREWS, 1990; BELLOCQ, 2000; SICK, 2001; BONVICINO; BEZERRA, 2003). Isto também foi verificado nas sete localidades aqui estudadas, nas quais as corujas foram registradas ocupando construções humanas em uma paisagem representada por um mosaico de áreas abertas e florestais. Em todas as sete amostras, os roedores representaram entre $97,3 \%$ e $99,1 \%$ do número mínimo de indivíduos de pequenos mamíferos, sendo Oligoryzomys sp. e Mus musculus os táxons mais frequentes.

Em áreas florestais alteradas e fragmentadas na Mata Atlântica, Oligoryzomys nigripes é uma espécie comum (FONSECA; KIERULFF, 1989; PARDINI; UMETSU, 2006), podendo ser a mais abundante, como registrado por Paglia et al. (1995) em três ambientes alterados (campo antrópico, capoeira e mata secundária) na Mata Atlântica do estado de Minas Gerais e por Dalmagro e Vieira (2005) para uma área de Floresta Ombrófila Mista no Rio Grande do Sul. No presente estudo, Oligoryzomys sp. foi o táxon mais frequente em cinco localidades, nas três regiões fitoecológicas (Floresta Ombrófila Mista e Densa, e Floresta Estacional Decidual). Isto pode estar associado aos padrões de uso 
de micro-habitat por Oligoryzomys. Dalmagro e Vieira (2005) verificaram uma preferência de $O$. nigripes por áreas em estágios iniciais de sucessão florestal, o que foi corroborado por Lima et al. (2010) e Melo et al. (2011). Outras espécies de Oligoryzomys também têm sido eventualmente registradas como o item mais abundante entre as presas de Tyto, como O. flavescens para o delta bonaerense e nos humidales de Iberá, na Argentina (MASSOIA; FORNES, 1964; PARDIÑAS et al., 2005), e Oligoryzomys sp. em Los Santiagos, um mosaico de áreas florestais e antropizadas no Equador (BRITO et al., 2015).

Mus musculus é uma espécie comum em habitações humanas e áreas agrícolas, onde tem sido registrada como a mais predada por Tyto furcata (e. g., SCHEIBLER; CHRISTOFF, 2007). Entre as amostras analisadas neste estudo, M. musculus foi a espécie mais frequente em duas localidades (Araquari e Quebra Queixo), que apresentam grande extensão de áreas agrícolas. Massoia (1989), Brito et al. (2015) e Lemos et al. (2015) também registraram esse roedor como um item muito comum em pelotas, respectivamente, em uma área urbana próxima de Buenos Aires, duas localidades antropizadas no Equador e uma área de restinga no Rio de Janeiro.

Marsupiais e roedores cricetídeos florestais (Brucepattersonius sp., Euryoryzomys russatus, Juliomys spp. e Sooretamys angouya) ocorreram em proporções menores, mas estiveram presentes nas amostras das sete localidades estudadas, enquanto caviomorfos e quirópteros ocorreram apenas em duas. Em geral, ocorrências similares também têm sido registradas em outros estudos na Mata Atlântica (SCHEIBLER; CHRISTOFF, 2007) e em outros biomas (GONZÁLEZ et al., 1995; ROCHA et al., 2011). No entanto, Souza et al. (2010) obtiveram maiores proporções de marsupiais e quirópteros em uma área de Mata Atlântica de Pernambuco; Bonvicino e Bezerra (2003) registraram Thylamys velutinus (Wagner, 1842) (Didelphidae) como a segunda espécie mais comum em uma localidade no Cerrado; e Cavia sp. é um item importante na dieta de Tyto na Bolívia (ALIAGA-ROSSEL; TARIFA, 2005). Essas diferenças demonstram como a dieta de Tyto pode variar em função da disponibilidade e vulnerabilidade das presas (BELLOCQ, 1998; SICK, 2001; BONVICINO; BEZERRA, 2003).

Na comparação entre as localidades analisadas neste estudo, as maiores riquezas de pequenos mamíferos foram registradas no interior (em Floresta Ombrófila Mista e Floresta Estacional Decidual) em relação ao litoral (em Floresta Ombrófila Densa). Isto pode estar associado a uma maior heterogeneidade ambiental nas localidades amostradas no interior, onde espécies de sigmodontinos típicos de ambientes abertos, alagados ou alterados foram exclusivamente registradas, como Bibimys sp., Calomys tener, Necromys lasiurus, Scapteromys meridionalis e Oxymycterus nasutus (ver PATTON et al., 2015).

Apesar de os egagropilos fornecerem uma grande quantidade de material para estudos com pequenos mamíferos, a identificação das espécies a partir desses fragmentos é uma tarefa difícil, principalmente em se tratando de ambientes com grande riqueza, como normalmente ocorre na região Neotropical (ANDERSON; LONG, 1961). Estudos mais aprofundados dos caracteres cranianos e dentários são necessários para uma melhor compreensão da variação morfológica intra e interespecíficas de várias espécies de pequenos mamíferos sul-americanos. Aliado a isto, a possibilidade de uso de análises moleculares para a identificação das espécies é outra abordagem útil (ROCHA et al., 2015). Identificações mais precisas, até a categoria de espécie, são fundamentais também para o refinamento das inferências paleoambientais feitas a partir de assembleias fósseis formadas pelo acúmulo de espécimes por predação de corujas, pois as espécies, tanto dos predadores quanto das presas, podem apresentar distribuições no passado distintas das atuais (e. g., PEARSON; PEARSON, 1993; HADLER et al., 2016; STUTZ et al., 2017).

\section{Agradecimentos}

Agradecemos a Alexandre T. Xavier, André L. Regolin, Artur Stanke, Elsimar S. da Silva, Ivo R. Ghizoni-Jr., Kaoey Machado, Levi K. Beckhauser, Marcos A. G. de Azevedo e Rosenei Olicheski pela coleta das pelotas e apoio em campo. A Edison A. Machado 
e Aline Possamai Della, pelo auxílio na preparação das amostras em laboratório. A ETS Energia, Transporte e Saneamento, Foz do Chapecó Energia S.A., Gehidro Engenharia e Consultoria Ltda., Maurique Consultores Associados e Sociambiental Consultores Associados Ltda. A dois avaliadores anônimos, cujas revisões contribuíram para maior clareza do texto. Ao CNPq (CNPq 444508/2014-7) (PH) e à Agencia Nacional de Promoción Científica y Tecnológica, Argentina (PICT 2014, \#1039) (UFJP) pelos auxílios concedidos.

\section{Referências}

ALIAGA-ROSSEL, E.; TARIFA, T. Cavia sp. como principal presa de la lechuza de campanario (Tyto alba) al final de la estación seca en una zona intervenida al norte del Departamento de La Paz, Bolivia. Ecología en Bolivia, La Paz, v. 40, n. 1, p. 35-42, 2005.

ANDERSON, S.; LONG, C. A. Small mammals in pellets of barn owls from Miñaca, Chihuahua. American Museum Novitates, New York, n. 2052, p. 1-3, 1961.

ANDREWS, P. Owls, caves and fossils. Chicago: University of Chicago Press, 1990. 232 p.

BARQUEZ, R. M. Breves comentarios sobre Molossus molossus (Chiroptera-Molossidae) de Bolivia. Historia Natural, Corrientes, v. 3, n. 18, p. 169-174, 1983.

BARQUEZ, R. M.; MARES, M. A.; BRAUN, J. K. The bats of Argentina. Special Publications, Museum of Texas Tech University, Lubbock, v. 42, p. 1-275, 1999.

BEISAW, A. M. Identifying and interpreting animal bones: a manual. College Station: Texas A \& M University Press, 2013. 214 p.

BELLOCQ, M. I. Prey selection by breeding and non-breeding barn owls in Argentina. The Auk, Albuquerque, v. 115, n. 1, p. 224-229, 1998.

BELLOCQ, M. I. A review of the trophic ecology of the Barn Owl in Argentina. Journal of Raptor Research, Albuquerque, v. 34, p. $108-119,2000$.

BONVICINO, C. R.; BEZERRA, A. M. R. Use of regurgitated pellets of Barn Owl (Tyto alba) for inventorying small mammals in the cerrado of Central Brazil. Studies on Neotropical Fauna and Environment, Tübingen, v. 38, n. 1, p. 1-5, 2003.

BORONI, N. L.; PARDIÑAS, U. F. J.; LESSA, G. Oligoryzomys fornesi (Massoia, 1973), Mammalia, Rodentia, Sigmodontinae: Distribution extension. Boletim do Museu de Biologia Mello Leitão, Santa Teresa, n. sér., v. 37, n. 3, p. 301-311, 2015.

BRITO, J.; ORELLANA-VÁSQUEZ, H.; CADENA-ORTIZ, H.; VARGAS, R.; POZO-ZAMORA, G. M.; CURAY, J. Mamíferos pequeños en la dieta de la lechuza Tyto alba (Strigiformes: Tytonidae) en dos localidades del occidente de Ecuador, con ampliación distribucional de Ichthyomys hydrobates (Rodentia: Cricetidae). Papéis Avulsos de Zoologia, São Paulo, v. 55, p. 261268, 2015.

CHARTER, M.; IZHAKI, I.; SHAPIRA, L.; LESHEM, Y. Diets of urban breeding barn owls (Tyto alba) in Tel Aviv, Israel.
Wilson Journal of Ornithology, Villanova, v. 119, n. 3, p. 484485, 2007.

CHEREM, J. J.; ALTHOFF, S. L.; REINICKE, R. C. Mamíferos. In: CHEREM, J. J.; KAMMERS, M. (Org.). A fauna das áreas de influência da Usina Hidrelétrica Quebra Queixo. Erechim: Habilis, 2008. p. 151-177.

CHEREM, J. J.; FERIGOLO, J. Descrição do sincrânio de Cavia aperea (Rodentia, Caviidae) e comparação com as demais espécies do gênero no Brasil. Papéis Avulsos de Zoologia, São Paulo, v. 52, p. 21-50, 2012.

CHRISTOFF, A. U.; VIEIRA, E. M.; OLIVEIRA, L. R.; GONÇALVES, J. W.; VALIATI, V. H.; TOMASI, P. S. A new species of Juliomys (Rodentia, Cricetidae, Sigmodontinae) from the Atlantic Forest of Southern Brazil. Journal of Mammalogy, Lawrence, v. 97, n. 5, p. 1469-1482, 2016.

DALMAGRO, A. D.; VIEIRA, E. M. Patterns of habitat utilization of small rodents in an area of Araucaria forest in Southern Brazil. Austral Ecology, Alice Springs, v. 30, p. 353-362, 2005.

DIAS, D. Sistemática molecular, biogeografia e diversificação de Brucepattersonius (Rodentia: Sigmodontinae). 2016. $72 \mathrm{f}$. Dissertação (Mestrado em Ciências Biológicas) - Universidade Federal do Espírito Santo, Vitória. 2016.

FONSECA, G. A. B.; KIERULFF, M. C. M. Biology and natural history of Brazilian Atlantic Forest small mammals. Bulletin of the Florida State Museum, Biological Sciences, Gainesville, v. 34, n. 3, p. 99-152, 1989.

FORMOSO, A. E.; TETA, P.; CARBAJO, A. E.; PARDIÑAS, U. F. J. Unraveling the patterns of small mammal species richness in the southernmost aridlands of South America. Journal of Arid Environments, London, v. 134, 136 e 144, 2016.

GARDNER, A. L. (Ed.). Mammals of South America. Vol. 1. Marsupials, xenarthrans, shrews, and bats. Chicago: University of Chicago Press, 2008. 669 p.

GOIN, F. J.; REY, P. Sobre las afinidades de Monodelphis Burnett, 1830 (Mammalia: Marsupialia: Didelphidae: Marmosinae). Neotropica, La Plata, v. 43, p. 93-98, 1997.

GONÇALVES, P. R.; MYERS, P.; VILELA, J. F.; OLIVEIRA, J. A. Systematics of species of the genus Akodon (Rodentia: Sigmodontinae) in Southeastern Brazil and implications for the biogeography of the campos de altitude. Miscellaneous Publications, Museum of Zoology, University of Michigan, Michigan, n. 197. p. 1-24, 2007.

GONZÁlEZ, E. M.; CLARAMUNT, S. J.; SARALEGUI, A. M. Mamíferos hallados en egagrópilas de Tyto alba (Aves, Strigiformes, Tytonidae) en Bagé, Rio Grande do Sul, Brasil. Iheringia, Série Zoologia, Porto Alegre, v. 86, p. 117-120, 1999.

GONZÁlEZ, E. M.; GONZÁlEZ, J.; FREGUEIRO, G.; SARALEGUI, A. Mamíferos encontrados en regurgitaciones de lechuzas del noreste de Uruguay (Mammalia: Rodentia: Marsupialia: Chiroptera). Comunicaciones Zoológicas del Museo de Historia Natural de Montevideo, Montevidéu, v. 12, n. 181, p. 1-4, 1995.

GOTELLI, N. J.; COLWELL, R. K. Estimating species richness. In: MAGURRAN, A. E.; MCGILL, B. J. (Ed.). Biological diversity: frontiers in measuring biodiversity. Oxford: Oxford University Press, 2011. p. 39-54. 
HADLER, P.; CHEREM, J. J.; TURBAY, R.; ALBERTI, A.; PARDIÑAS, U. F. J. Diversidade de pequenos mamíferos (Didelphimorphia e Rodentia) do Holoceno do nordeste do estado do Rio Grande Do Sul, Brasil: implicações taxonômicas e paleoambientais. Revista Brasileira de Paleontologia, Porto Alegre, v. 19, p. 127-144, 2016.

HEISLER, L. M., SOMERS, C. M., POULIN, R. G. Owl pellets: a more effective alternative to conventional trapping for broad-scale studies of small mammal communities. Methods in Ecology and Evolution, London, v. 7, p. 96-103, 2016.

IUCN. IUCN red list of threatened species. Version 2017.1. 2017. Disponível em: <http://www.iucnredlist.org>. Acesso em: 18 jul. 2017.

LANGGUTH, A. Las especies uruguayas del genero Oryzomys. Comunicaciones Zoológicas del Museo de Historia Natural de Montevideo, Montevidéu, v. 7, p. 1-19, 1963.

LEKUNZE, M. L.; EZEALOR, U. A.; AKEN'OVA, T. Prey groups in the pellets of the barn owl Tyto alba (Scopoli) in the Nigerian savanna. African Journal of Ecology, Oxford, v. 39, p. 28-44, 2001 .

LEMOS, H. M.; SILVA, C. A. O.; PATIU, F. M.; GONÇALVES, P. R. Barn Owl pellets (Aves: Tyto furcata) reveal a higher mammalian richness in the Restinga de Jurubatiba National Park, Southeastern Brazil. Biota Neotropica, Campinas, v. 15, n. 2, e20140121, 2015.

LEITE, P. F. Contribuição ao conhecimento fitoecológico do sul do Brasil. Ciência e Ambiente, Santa Maria, v. 24, p. 51-73, 2002. LIMA, D. O.; AZAMBUJA, B. O.; CAMILOTTI, V. L.; CÁCERES, N. C. Small mammal community structure and microhabitat use in the austral boundary of the Atlantic Forest, Brazil. Zoologia, Curitiba, v. 27, n. 1, p. 99-105, 2010.

MACHADO, L. F.; PARESQUE, R.; CHRISTOFF, A. U. Anatomia comparada e morfometria de Oligoryzomys nigripes e O. flavescens (Rodentia, Sigmodontinae) no Rio Grande do Sul, Brasil. Papéis Avulsos de Zoologia, São Paulo, v. 51, n. 3, p. 29-47, 2011.

MASSOIA, E. Oxymycterus iheringi (Rodentia-Cricetidae) nueva especie para la Argentina. Physis, Buenos Aires, v. 24, n. 67, p. 129-136, 1963.

MASSOIA, E. La alimentación de algunas aves del orden Strigiformes en la Argentina. Hornero, Buenos Aires, v. 12, n. 1 (extra), p. 125-148, 1983.

MASSOIA, E. Animales depredados por Tyto alba tuidara en la Ciudad de San Miguel, partido de General Sarmiento, provincia de Buenos Aires. Boletín Científico APRONA, Buenos Aires, v. 15, p. 2-7, 1989.

MASSOIA, E.; FORNES, A. Pequeños mamíferos (Marsupialia, Chiroptera y Rodentia) y aves obtenidos en regurgitaciones de lechuzas (Strigiformes) del delta Bonaerense. Delta do Paraná, Investigaciones Agrícolas, Buenos Aires, v. 4, n. 6, p. 27-34, 1964.

MASSOIA, E.; FORNES, A. Claves para el reconocimiento de los roedores del Delta del Paraná (Mammalia). Revista del Instituto de Investigaciones Agropecuarias, Buenos Aires, v. 253, p. 11-18, 1969.

MEEK, W. R.; BURMAN, P. J.; SPARKS, T. H.; NOWAKOWSKI, M.; BURMAN, N. J. The use of Barn Owl Tyto alba pellets to assess population change in small mammals. Bird Study, Irving, v. 59, n. 2 , p. $166-174,2012$.
MELO, G.; SPONCHIADO, J.; MACHADO. A.; CÁCERES, N. Small-mammal community structure in a South American deciduous Atlantic Forest. Community Ecology, Budapest, v. 12, n. 1, p. 58-66, 2011.

MIRANDA, J. M. D.; BERNARDI, I. P.; PASSOS, F. C. Chave ilustrada para determinação dos morcegos da Região Sul do Brasil. Curitiba: João M. D. Miranda, 2011. 51 p.

MMA. Shapefile do mapa de vegetação do Brasil. 1992. Disponível em: <http://mapas.mma.gov.br/i3geo/datadowload. htm>. Acesso em: 17 abr. 2018

MMA. Lista das espécies da fauna brasileira ameaçadas de extinção. 2014. Disponível em: <www.icmbio.gov.br/portal/ biodiversidade/faunabrasileira/lista-de-especies.html>. Acesso em: 19 dez. 2014.

MOOJEN, J. Os roedores do Brasil. Rio de Janeiro: Instituto Nacional do Livro, 1952. 214 p.

MORENO, E., BARBOSA, A. Distribution patterns of small mammal fauna along gradients of latitude and elevation in Northern Spain. Zeitschrieft für Saügetierkd, Berlin, v. 57, p. 169-175, 1992.

MOTTA-JUNIOR, J. C.; TALAMONI, S. A. Biomassa de presas consumidas por Tyto alba (Strigiformes: Tytonidae) durante a estação reprodutiva no Distrito Federal. Ararajuba, Santa Cruz do Sul, v. 4, n. 2, p. 38-41, 1996.

MUSSER, G. G. Results of the Archbold Expeditions. No 105. Notes on systematics of Indo-Malayan murid rodents and descriptions of new genera and species from Ceylon, Sulawesi, and the Philippines. Bulletin of the American Museum of Natural History, New York, v. 168, n. 3, p. 225-334, 1981

OLIVEIRA, J. A.; GONÇALVES, P. R. Genus Oxymycterus Waterhouse, 1837. In: PATTON, J. L.; PARDINÃS, U. F. J.; D'ELÍA, G. (Ed.). Mammals of South America. Vol. 2. Rodents. Chicago: University of Chicago Press, 2015. p. 247-268.

PAGLIA, A. P.; MARCO JR., P.; COSTA, F. M.; PEREIRA, R. F.; LESSA, G. Heterogeneidade estrutural e diversidade de pequenos mamíferos em um fragmento de mata secundária de Minas Gerais, Brasil. Revista Brasileira de Zoologia, Curitiba, v. 12, n. 1, p. $67-$ 79, 1995.

PARANÁ. Lista das espécies de mamíferos ameaçados no estado do Paraná e suas respectivas categorias de ameaça. Anexo a que se refere o decreto $\mathrm{n}^{\mathrm{0}}$ 7264/2010.

PARANÁ. Portaria IAP no 59, de 15 de abril de 2015. Disponível em: <http://celepar7.pr.gov.br/sia/atosnormativos/form_cons_ato1. asp?Codigo $=2921>$. Acesso em: 22 ago. 2015.

PARDIÑAS, U. F. J.; TETA, P.; D’ELÍA, G.; GALLIARI, C. Rediscovery of Juliomys pictipes (Rodentia: Cricetidae) in Argentina: emended diagnosis, geographic distribution, and insights on genetic structure. Zootaxa, Auckland, n. 1758, p. 2944, 2008

PARDIÑAS, U. F. J.; TETA, P.; HEINONEN FORTABAT, S. Vertebrate prey of the barn owl (Tyto alba) in subtropical wetlands of northeastern Argentina and eastern Paraguay. Journal of Raptor Research, Albuquerque, v. 39, n. 1, p. 65-69, 2005.

PARDIÑAS, U. F. J.; VOGLINO, D.; GALLIARI, C. A. Miscellany on Bibimys (Rodentia, Sigmodontinae), a unique akodontine cricetid. Mastozoología Neotropical, Mendoza, v. 24, n. 1, p. 241 250, 2017. 
PARDINI, R.; UMETSU, F. Pequenos mamíferos não voadores da Reserva Florestal do Morro Grande - distribuição das espécies e da diversidade em uma área de Mata Atlântica. Biota Neotropica, Campinas, v. 6, n. 2, p. 1-22, 2006.

PASSOS, F. C.; MIRANDA, J. M. D.; BERNARDI, I. P.; KANUOLIVIERA, N. Y.; MUNSTER, L. C. Morcegos da Região Sul do Brasil: análise comparativa da riqueza de espécies, novos registros e atualizações nomenclaturais (Mammalia, Chiroptera). Iheringia, Série Zoologia, Porto Alegre, v. 100, n. 1, p. 25-34, 2010.

PATTON, J. L.; PARDINÃS, U. F. J.; D’ELÍA, G. Mammals of South America. Vol. 2. Rodents. Chicago: University of Chicago Press, 2015. 1336 p.

PAVAN, S. E.; VOSS, R. S. A revised subgeneric classification of short-tailed opossums (Didelphidae: Monodelphis). American Museum Novitates, New York, n. 3868, p. 1-44, 2016.

PEARSON, O. P.; PEARSON, A. K. La fauna de mamíferos pequeños cerca de Cueva Traful I, Argentina: pasado y presente. Præhistoria, Buenos Aires, v. 1, p. 73-89, 1993.

PEÇANHA, W. T.; GONÇALVES, G. L.; ALTHOFF, S. L.; FREITAS, T. R. O.; HASS, I. Range extension of the Atlantic Forest Hocicudo, Oxymycterus dasytrichus (Schinz, 1821), to the state of Santa Catarina, southern Brazil. Check List, Rio Claro, v. 12, n. 1847, p. 1-7, 2016.

PETERS, F. G.; ROTH, P. R. O.; CHRISTOFF, A. U. Primeiro registro documentado de Holochilus brasiliensis (Desmarest, 1819) e Calomys laucha (G. Fischer, 1914) no estado de Santa Catarina, sul Brasil. Biotemas, Florianópolis, v. 26, n. 3, p. 177-184, 2013.

QUINTElA, F. M.; GONÇALVES, G. L.; ALTHOFF, S. L.; SBALQUEIRO, I. J.; OLIVEIRA, L. F. B.; FREITAS, T. R. O. A new species of swamp rat of the genus Scapteromys Waterhouse, 1837 (Rodentia: Sigmodontinae) endemic to Araucaria angustifolia forest in southern Brazil. Zootaxa, Auckland, v. 3811, n. 2, p. $207-$ 225,2014

RIO GRANDE DO SUL. Portaria SEMA no 79 , de 31 de outubro de 2013. Disponível em: <https://www.legisweb.com.br/ legislacao/?id=261368> . Acesso em: 18 jul. 2017.

RIO GRANDE DO SUL. Decreto no 51.797, de 8 de setembro de 2014. Diário Oficial.

ROCHA, R. G.; FERREIRA, E.; LEITE, Y. L.; FONSECA, C.; COSTA, L. P. Small mammals in the diet of barn owls, Tyto alba (Aves: Strigiformes) along the mid-Araguaia river in central Brazil. Zoologia, Curitiba, v. 28, n. 6, p. 709-716, 2011.

ROCHA, R. G.; JUSTINO, J.; LEITE, Y. L.; FONSECA, C.; COSTA, L. P. DNA from owl pellet bones uncovers hidden biodiversity, Systematics and Biodiversity, London, v. 13, n. 4, p. 403-412, 2015.

SALAZAR-BRAVO, J. Genus Calomys Waterhouse, 1837. In: PATTON, J. L.; PARDINÃS, U. F. J.; D’ELÍA, G. (Ed.). Mammals of South America. Vol. 2. Rodents. Chicago: University of Chicago Press, 2015. p. 481-507.
SANTA CATARINA. Resolução Consema no 2, de 6 de dezembro de 2011. Disponível em: <http://media.wix.com/ugd/4e96b1_7aee 52da52ca9f5b16f823476a0b1cfc.pdf>. Acesso em: 11 nov. 2013.

SANTA CATARINA. Resolução Consema no ${ }^{-}$, de 14 de setembro de 2012. Disponível em: <www.fatma.sc.gov.br/upload/ rppne/resconsema201208.pdf $>$. Acesso em: 22 ago. 2015.

SCHEIBLER, D. R.; CHRISTOFF, A. U. Habitat associations of small mammals in southern Brazil and use of regurgitated pellets of birds of prey for inventorying a local fauna. Brazilian Journal of Biology, São Carlos, v. 67, n. 4, p. 619-625, 2007.

SICK, H. Ornitologia brasileira. Rio de Janeiro: Nova Fronteira, 2001. 912 p.

SOUZA, D. P.; ASFORA, P. H.; LIRA, T. C.; ASTÚA, D. Small mammals in Barn Owl (Tyto alba - Aves, Strigiformes) pellets from northeastern Brazil, with new records of Gracilinanus and Cryptonanus (Didelphimorphia, Didelphidae). Mammalian Biology, Jena, v. 75, n. 4, p. 370-374, 2010.

STUTZ, N. S.; CHEREM, J. J.; PARDIÑAS, U. F. J.; HADLER, P. Roedores sigmodontíneos (Mammalia, Rodentia, Cricetidae) Holocênicos do Rio Grande do Sul, Brasil - O sítio RSTQ-58: Afonso Garivaldino Rodrigues. Revista Brasileira de Paleontologia, Porto Alegre, v. 20, n. 1, p. 133-148, 2017.

TAYLOR, I. Barn Owls. Cambridge: University Press, 1994. $320 \mathrm{p}$.

TETA，P.; GONZÁLEZ-FISCHER，C. M.; CODESIDO, M.; BILENCA, D. N. A contribution from Barn Owl pellets analysis to known micromammalian distributions in Buenos Aires province, Argentina. Mammalia, Paris, v. 74, p. 97-103, 2010.

TORRE, I.; ARRIZABALAGA, A.; CARLES, F. Three methods for assessing richness and composition of small mammal communities. Journal of Mammalogy, Lawrence, v. 85, p. 524-530, 2004.

VILELA J. F.; GONÇALVES, P. R.; OLIVEIRA, J. A. Genus Brucepattersonius Hershkovitz, 1998. In: PATTON, J. L.; PARDINÃS, U. F. J.; D'ELÍA, G. (Ed.). Mammals of South America. Vol. 2. Rodents. Chicago: University of Chicago Press, 2015. p. 211-507.

VOGLINO, D.; PARDIÑAS, U. F. J.; TETA, P. Holochilus chacarius chacarius (Rodentia, Cricetidae) en la Provincia de Buenos Aires, Argentina Mastozoología Neotropical, Mendoza, v. 11, n. 2, p. 243-247, 2004.

VOSS, R. S.; JANSA, S. A. Phylogenetic relationships and classification of didelphid marsupials, an extant radiation of New World metatherian mammals. Bulletin of the American Museum of Natural History, New York, v. 322, p. 1-177, 2009.

WILSON, D. E.; REEDER, D. M (Ed.). Mammal species of the world: a taxonomic and geographic reference. 3. ed. Baltimore: The Johns Hopkins University Press, 2005. 2142 p.

XIMENEZ,A. Notas sobre el género Cavia Pallas con la descripción de Cavia magna sp. n. (Mammalia - Caviidae). Revista Nordestina de Biologia, João Pessoa, v. 3, n. especial, p. 145-179, 1980. 


\section{ANEXO I}

Lista dos espécimes depositados na Coleção de Mamíferos do Departamento de Ecologia e Zoologia da Universidade Federal de Santa Catarina (UFSC) utilizados na comparação com os espécimes presentes nos egagropilos.

Cryptonanus guahybae: UFSC 4465, 4589, 4854, 5197, 5234, 5434. Gracilinanus microtarsus: UFSC 3851, 4857, 5951, 5893. Marmosa paraguayana: UFSC 4048, 4374. Monodelphis dimidiata: UFSC 3778, 4823, 4861, 5740. Monodelphis iheringi: UFSC 3797, 4585, 4607, 5436. Monodelphis scalops: UFSC 3780, 3915, 4056. Akodon montensis: UFSC 222, 288, 3481, 3701, 3702, 3804, 4068, 4073, 4683, 5090-5096, 5098-5101, 5103, 5104, 5109, 5110, 5628. Akodon paranaensis: UFSC 3628, 3631, 3721, 4064, 4086, 4686, 5138, 5154, 5160, 5161, 5627, 5685. Brucepattersonius iheringi: UFSC 3425, 4925. Brucepattersonius soricinus: UFSC 4692, 4810. Calomys tener: UFSC 5166, 5490.

Euryoryzomys russatus: UFSC 4960, 4961. Juliomys ossitenuis: UFSC 950, 4812. Juliomys pictipes: UFSC 2960, 2963. Necromys lasiurus: UFSC 3599, 3600, 3876, 4714. Nectomys squamipes: UFSC 5078, 5494. Oligoryzomys flavescens: UFSC 60, 3643, 4133, 4138, 4400, 4548, 4694, 4695, 5036, 5043, 5048-5050, 5639, 5891. Oligoryzomys nigripes: UFSC 498, 501, 502, 510, 3638, 4537-4540, 4544-4547, 5357, 5640, 5641, 5736, 5837, 5838. Oxymycterus dasythrichus: UFSC 2084, 2085, 2086. Oxymycterus nasutus: UFSC 955, 5892, 5934. Oxymycterus quaestor: UFSC 4817, 5645, 5787. Scapteromys meridionalis: UFSC 2662, 2671, 3597, 3819. Sooretamys angouya: UFSC 5927, 6031. Rattus norvegicus: UFSC 428, 5456. Rattus rattus: UFSC 5565 , 5749. Cavia aperea: UFSC 3054, 3055, 3089. Cavia fulgida: UFSC 2398, 2399. Euryzygomatomys spinosus: UFSC 73, 2931, 4005. 